\title{
Quality of Life in Children with Disorders of Sex Development
}

\author{
Nalini M Selveindran ${ }^{a}$ Syed Zulkifli Syed Zakaria ${ }^{b} \quad$ Muhammad Yazid Jalaludin ${ }^{c}$ \\ Rahmah Rasat ${ }^{\text {b }}$ \\ a Department of Paediatrics, Hospital Putrajaya,, Putrajaya, Malaysia; ${ }^{b}$ Department of Paediatrics, University \\ Kebangsaan Malaya Medical Centre, Kuala Lumpur, Malaysia; ' Department of Paediatrics, Faculty of Medicine, \\ University Malaya, Kuala Lumpur, Malaysia
}

\author{
Keywords \\ Disorders of sex development · Quality of life · Children · \\ PedsQL questionnaire
}

\begin{abstract}
Background/Aims: Disorders of sex development (DSD) are a heterogeneous group of rare conditions. Evidence-based treatment is challenged by a lack of clinical longitudinal outcome studies. We sought to investigate the quality of life of children with DSD other than congenital adrenal hyperplasia. Methods: The participants (aged 6-18 years) were 23 patients raised as males and 7 patients raised as females. Control data were obtained from representatives of the patients' siblings matched for age and gender. The Pediatric Quality of Life Inventory ${ }^{\mathrm{TM}}$ Version 4.0 (PedsQL) Generic Core Scales were used as the study tool. Results: In comparison with the reference data, the patient group had significantly lower overall PedsQL $(p<0.01)$ and school functioning $(p<0.01)$ scores. Also, the total PedsQL score was significantly lower in patients with DSD who were of female social sex as compared to the controls who were females. Family income, surgical procedures, degree of virilization, and mode of puberty did not influence the PedsQL scores. Conclusion: This study revealed a poorer quality of life for patients with DSD as com-
\end{abstract}

pared to the age-matched control group. This highlights the need for a skilled multidisciplinary team to manage this group of patients.

(c) 2017 S. Karger AG, Basel

\section{Introduction}

Disorders of sex development (DSD) are a heterogeneous group of rare conditions which has an incidence rate of approximately 1 in 4,500-5,500 [1]. However, more specific conditions occur at a rate of $1: 100,000$ births or fewer [2]. DSD are an umbrella term for a variety of conditions that encompass various aetiologies and presentations.

The long-term psychological, physical, and social outcomes for young people with DSD are unclear. Recent reports have raised concerns regarding early corrective surgery and decisions regarding sex of rearing, which are matched by concerns expressed by patient advocacy groups $[3,4]$. Studies on this group of patients have usually focused on psychosexual and surgical outcomes and have rarely considered overall quality of life outcomes [5-8]. Nevertheless, health-related quality of life measures are an adequate endpoint by which to evaluate out- 
comes in this group of patients because they take into account multiple dimensions of the patient's wellbeing [9].

Most research in this area has focused on patients with congenital adrenal hyperplasia $(\mathrm{CAH})$, with very few studies concentrating on other DSD [10]. Unfortunately, patients with DSD other than CAH report the lowest satisfaction with medical care [11]. The management principles for this group of patients are usually less clear and are often extrapolated from data on CAH patients [5]. Given the fact that the diagnoses of DSD encompass a wide variety of conditions this is less than optimal. The management of patients with DSD is also influenced by the sociocultural and religious environment [12-14]. Most studies have focused on CAH in a Caucasian population. We therefore evaluated psychosocial outcomes in patients with DSD other than CAH in our particular context of an Asian, predominantly Muslim culture.

\section{Research Objectives}

The general objective of this study was to evaluate the quality of life of patients with DSD other than CAH. In addition, we also sought to determine whether family income, degree of virilization at birth, mode of puberty, and number of surgeries had an influence on the quality of life scores.

\section{Materials and Methods}

\section{Study Outline}

The study was conducted from 1 November 2013 to 30 November 2014. Individuals aged between 6 and 18 years $(n=45)$ with DSD other than CAH who attended the paediatric endocrine clinic at the University Kebangsaan Malaysia Medical Centre (UKMMC) and University Malaya Medical Centre (UMMC) were invited to participate in the study. These two centres are the largest and oldest paediatric endocrine facilities in the country. Exclusion criteria were those with other syndromes, and psychiatric and neurologic disorders $(n=5)$. Children whose parents did not attend the follow-up despite reminders were also excluded $(n=10)$. The final sample consisted of 30 subjects: male social sex, $n=23$ (XY = $21, X X=2)$ and female social sex, $n=7(X Y=6, X X=1)$. The control group consisted of a representative from each of the patient's siblings $(n=30$ : males $=22$, females $=8)$.

Written informed consent was obtained during a clinic visit from the parents or legal guardians of all the patients before participation. Consenting participants received a questionnaire booklet that they were asked to complete by the end of the clinic visit. The study protocol was approved by the ethical committees of UKMMC and UMMC. The study protocol conformed to the ethical standards in the Helsinki Declaration II.

Background clinical data obtained from medical records included age at diagnosis, karyotype, gender assigned, clinical and biochemical data at presentation including degree of virilization, number and nature of surgical procedures performed, and pubertal status. Current information on the patients, physical examination, pubertal development, complications, medication, and socioeconomic data was obtained at the clinic interview.

We decided to group the patients by karyotype and sex of rearing/recent gender, a classification system previously used by the German Network of Disorders of Sex Development (DSD)/Intersexuality and also suggested by the Consensus Statement of the ESPE/LWSPE Conference $[15,16]$. The resulting subgroups were defined as follows:

Subgroup 1 (XX-F, $n=1)$

Individuals with $\mathrm{XX}$ karyotype and reared as girls/living as women.

\section{Subgroup $2(\mathrm{XX}-\mathrm{M}, n=2)$}

Individuals with $\mathrm{XX}$ karyotype and reared as boys/living as boys. This subgroup included patients diagnosed with ovotesticular DSD and XX sex reversal.

Subgroup 3 (XY-F, $n=6$ )

Individuals with $\mathrm{XY}$ karyotype and reared as girls/living as women. This subgroup included patients with partial androgen insensitivity syndrome, ovotesticular DSD, and gonadal dysgenesis.

\section{Subgroup $4(\mathrm{XY}-\mathrm{M}, n=21)$}

Individuals with XY karyotype and reared as boys/living as men. This subgroup included patients with partial androgen insensitivity syndrome, ovotesticular DSD, gonadal dysgenesis, and hypospadias.

\section{Questionnaire}

The study tool used was The Pediatric Quality of Life Invento$\mathrm{ry}^{\mathrm{TM}}$ Version 4.0 (PedsQL) Generic Core Scales questionnaire [17, 18]. The UK English and the Malaysian language translations were used as appropriate.

The PedsQL questionnaire seeks to measure the following core dimensions of health: physical function, social function, emotional function, and school function through the answers given to 23 questions. The parent proxy version was used, and the questionnaire was also age appropriate with a modified version for each age group (5-7, 8-12, 13-18 years). For each question the parent was asked to score the answer on a scale of $0-4$. Subsequently, this score was linearly transformed into a $0-100$ scale so that a higher score would indicate a better quality of life $(0-100,1-75,2-50,3-25$, 4-0). To create scores for each dimension, the mean of the scores was computed, and here also a higher score indicated a better quality of life in each of the four dimensions.

\section{Statistical Analysis}

Data were analyzed using IBM SPSS version 22. Statistical difference was assessed using a $t$ test for parametric data and the Mann-Whitney and Kruskal-Wallis tests for non-parametric data. For categorical data, the $\chi^{2}$ test was used. For identification of the risk factors, bivariate analysis (Spearman's rank-order correlation) was performed. Statistical significance was assumed when $p<0.05$; however, this was adjusted using the Bonferroni-Holm method to account for multiple comparisons. 
Table 1. Sociodemographic characteristics of cases and controls

\begin{tabular}{|c|c|c|c|}
\hline Variable & Cases & Controls & $p$ value \\
\hline Mean age at study entry $( \pm S D)$, years ${ }^{a}$ & $13(3.3)$ & $12.8(2.5)$ & 0.76 \\
\hline \multicolumn{4}{|l|}{ Gender frequency, $n(\%)^{\mathrm{b}}$} \\
\hline Male & $23(76.7)$ & $22(73.3)$ & 0.77 \\
\hline Female & $7(23.3)$ & $8(27.7)$ & \\
\hline Mean family income (SD), MYR ${ }^{\mathrm{a}}$ & $3,850(1,365)$ & $4,033(1,419)$ & 0.80 \\
\hline \multicolumn{4}{|l|}{ Parental marital status, $n^{\mathrm{c}}$} \\
\hline Married & 29 & 29 & 1.00 \\
\hline Single & 1 & 1 & \\
\hline
\end{tabular}

${ }^{\mathrm{a}}$ Independent $t$ test; ${ }^{\mathrm{b}} \chi^{2}$ test; ${ }^{\mathrm{c}}$ Fisher's exact test.
Table 2. Baseline characteristics of DSD cases

\begin{tabular}{lll}
\hline & Male $(n=23)$ & Female $(n=7)$ \\
\hline $\begin{array}{l}\text { Mean age at study entry }( \pm S D), \\
\text { years }\end{array}$ & & $12.5(3.4)$ \\
Prader score, $n(\%)$ & 0 & \\
$\quad 2$ & $2(9)$ & $2(28)$ \\
3 & $15(65)$ & \\
4 & $6(26)$ & \\
5 & $18(78.3)$ & $6(85.7)$ \\
Surgical procedures, $n(\%)^{\mathrm{a}}$ & & \\
Number of surgical procedures & 8 & 6 \\
$\quad \leq 2$ & 10 & 0 \\
$\quad>2$ & 6 & 5 \\
Gonadectomy, $n$ & &
\end{tabular}

DSD, disorders of sex development. ${ }^{\text {a Frequency. }}$

\section{Results}

\section{Patient Cohort and Control}

There was no significant difference between the case and control groups with regard to mean age at study entry, gender distribution, mean family income, and parental marital status (Table 1). Similarly, there was no difference between male and female patients as compared to male and female controls with regard to the above demographic characteristics.

Patient characteristics are summarized in Tables 1-3. Twenty-one patients were included in the group XY-M with a median age of 14 years [10-15], while 6 patients were included in the group XY-F with a median age of 14.5 years (12.8-18). There was 1 patient in the XX-F group who was 12 years old and 2 patients in the XX-M group aged 11 and 13. Nineteen patients (63.3\%) were
Table 3. Groupings of patients and distribution of diagnoses

\begin{tabular}{lrrrrrr}
\hline \multirow{2}{*}{ Diagnosis } & \multicolumn{2}{l}{ Groups } & & \multirow{2}{*}{ Total } \\
\cline { 2 - 4 } & XY-M & XY-F & XX-F & XX-M & \\
\hline Gonadal dysgenesis & 13 & 0 & 0 & 0 & 13 \\
Mixed gonadal dysgenesis & 3 & 1 & 0 & 0 & 4 \\
Androgen insensitivity & 1 & 5 & 0 & 0 & 6 \\
Ovotestis & 3 & 0 & 1 & 1 & 5 \\
5a-Reductase deficiency & 1 & 0 & 0 & 0 & 1 \\
Sex reversal & 0 & 0 & 0 & 1 & 1 \\
\hline Total & 21 & 6 & 1 & 2 & 30 \\
\hline
\end{tabular}

adolescents (more than 12 years old). Only 5 (16.7\%) patients had gone into spontaneous puberty at the time of study, with a mean age of 11.8 years, and all of them were male.

The majority of the patients had undergone one or more surgical procedures, with $24(70.8 \%)$ having had a genital reconstruction. Eleven patients had gonadectomy, in 9 cases bilateral. Two patients had gonadoblastoma at the time of the removal of gonads. Two patients who had a diagnosis of ovotesticular DSD underwent gender change during the follow-up period of this study.

\section{PedsQL Scores}

In comparison with the control group, the patient group had significantly lower overall PedsQL $(p<0.01)$ and school functioning $(p<0.01)$ scores (Table 4$)$. A comparison of the total PedsQL score between the subgroups revealed no significant group differences, with slightly lower non-significant scores in the XY-F group as compared to the XY-M group (Table 5). Further analysis revealed that the total PedsQL score was significantly 
Table 4. Total and subscale PedsQL scores (physical, emotional, social, and schooling) in patients with DSD as compared with the control group

\begin{tabular}{llll}
\hline & Cases & Controls & $p$ value \\
\hline Physical & $100(21.9)$ & $100(6.2)$ & 0.163 \\
Emotional & $90(32.5)$ & $100(12.5)$ & 0.063 \\
Social & $95(20)$ & $100(5)$ & 0.031 \\
Schooling & $85(35)$ & $100(11.3)$ & 0.003 \\
\hline Total $^{\mathrm{a}}$ & $90.6(24.2)$ & $100(13.2)$ & 0.004 \\
\hline
\end{tabular}

A Bonferroni-adjusted alpha level of 0.01 was used to account for multiple comparisons. Scores are given as median (IQR), with lower scores denoting poorer outcomes. PedsQL, Pediatric Quality of Life Inventory ${ }^{\mathrm{TM}}$ Version 4.0; DSD, disorders of sex development. ${ }^{a}$ Denotes significant variables.

Table 6. Total and subscale PedsQL scores (physical, emotional, social, and schooling) in female patients with DSD as compared with the female control group

\begin{tabular}{llll}
\hline & Cases & Controls & $p$ value \\
\hline Physical & $81.3(37.5)$ & $100(7.8)$ & 0.19 \\
Emotional & $95(45)$ & $100(3.8)$ & 0.19 \\
Social & $95(30)$ & $100(0)$ & 0.07 \\
Schooling & $75(40)$ & $100(7.5)$ & 0.014 \\
\hline Total $^{\text {a }}$ & $86.3(31.3)$ & $100(4.8)$ & 0.004 \\
\hline
\end{tabular}

A Bonferroni-adjusted alpha level of 0.01 was used to account for multiple comparisons. Scores are given as median (IQR), with lower scores denoting poorer outcomes. PedsQL, Pediatric Quality of Life Inventory ${ }^{\mathrm{TM}}$ Version 4.0; DSD, disorders of sex development. ${ }^{a}$ Denotes significant variables.

lower in patients with DSD who were of female social sex as compared to the controls who were females (Table 6). This trend was not seen in the male patients' scores.

\section{Associations}

Our analysis found that there was no association between family income $(r=0.075, p=0.57)$ and the total PedsQL scores. As for the effect of repeated surgeries on the total scores, there was no significant correlation $(r=$ $0.058, p=0.76$ ). There was also no significant correlation between the degree of virilization at diagnosis and the total PedsQL scores for the population XY-F $(r=0.41, p=$ $0.41)$ and XY-M $(r=0.19, p=0.42)$. To evaluate the effect of the mode of puberty on quality of life, the PedsQL
Table 5. Total and subscale PedsQL scores (physical, emotional, social, and schooling) in patients with DSD with XY-M as compared with XY-F

\begin{tabular}{lrll}
\hline & XY-M & XY-F & $p$ value \\
\hline Physical & $100(18.8)$ & $78.2(38.3)$ & 0.26 \\
Emotional & $85(33.8)$ & $82.5(50)$ & 0.76 \\
Social & $95(13.8)$ & $85(33.8)$ & 0.48 \\
Schooling & $85(28.8)$ & $65(42.5)$ & 0.27 \\
\hline Total & $88.4(21.2)$ & $78.3(30.5)$ & 0.32 \\
\hline
\end{tabular}

A Bonferroni-adjusted alpha levels of 0.01 was used to account for multiple comparisons. Scores are given as median (IQR), with lower scores denoting poorer outcomes. PedsQL, Pediatric Quality of Life Inventory ${ }^{\mathrm{TM}}$ Version 4.0; DSD, disorders of sex development.

scores of those who had spontaneous puberty were compared with those of the group who underwent induction of puberty. The results indicated that there was no significant difference between the groups.

\section{Discussion}

Health-related quality of life scores are important indicators of therapeutic outcomes in chronic conditions $[19,20]$. In patients with DSD, these scores can give an indication as to whether medical intervention has had a positive impact on their wellbeing [11]. The present study revealed an overall impaired quality of life in the patient group, especially in the schooling dimension. These findings are in agreement with other studies that found a reduction in the quality of life of patients with DSD [21]. The results of a study on children with DSD conducted by Jürgensen et al. [22] found a reduction in quality of life in the schooling, physical wellbeing, and self-esteem dimensions.

We hypothesized that repeated surgeries would be associated with reduced quality of life. However, according to our results, there was no significant association between the number of surgeries and PedsQL scores. Jürgensen et al. [22] in their study of health quality of life in children with DSD did not find a similar association either. However, they caution that this aspect should be reevaluated in adulthood when the implications of surgical repair or gonadectomy may be more obvious. We also did not find a significant association between mode of puberty (spontaneous vs. induced) and quality of life. In ad- 
olescence, delayed onset of puberty, hormonal changes, and abnormalities of external genitalia may be a significant stressor. Follow-up studies may need to be performed to evaluate this association more fully. Given the low numbers and the fact that the study questionnaire was filled out by the parents, we may not be able to demonstrate significant pathology.

A variety of other factors may contribute to the impaired quality of life in children with DSD. Societal and peer pressure on the child and the family, parental support, and the patient's own personality are factors that should be accounted for [23, 24]. In order to comprehensively assess quality of life we would have to evaluate relationships, family dynamics, and the patients' own perceptions by using quantitative methods of analysis [5]. Moreover, there are added difficulties given the fact that these patients live in a developing country. Poverty, ignorance, lack of accessibility to specialized medical care, and lack of psychological services may all contribute negatively to these patients' outcomes [25].

The subgroup analysis aimed to test the hypothesis that there would be a significant difference among those with the XY karyotype who had a male social sex as compared to a female social sex. Although not of statistical significance, the XY-F group had consistently poorer scores. The effect of prenatal androgens on gender behaviour is well established because virilization may have an effect on self-image and lead to negative social reactions $[11,23]$. However, we did not find a significant correlation between the degree of virilization and the Ped$s Q L$ scores. The XY-F group may be an at-risk group that will need further follow-up in adulthood. We also demonstrated that it was particularly female patients with DSD who had significantly lower quality of life scores as compared with the control group. In an Asian culture, women are more subject to blame in issues related to marriage and fertility [26]. Thus, this is a group that needs greater attention from an experienced DSD team. We are also cognisant of the fact that the quality of life scores obtained in childhood may not correspond to those obtained in adulthood. The children may not have fully realized the implication of having this diagnosis in the realms of sexual relationships, life partners, marriage, and fertility. We did not evaluate sexual function as our cohort was too young, although this may influence the overall quality of life in adulthood $[5,27,28]$. However, this score in childhood can be used to identify at-risk groups that may need counselling in adulthood [29]. It is of paramount importance then that mechanisms for transition care are put into place in an orderly fashion

Impaired Quality of Life in Children with Disorders of Sex Development and that patients are given an increasing role to play in decision-making.

Our study does have some limitations. First, the questionnaire was not specific for the DSD conditions assessed. Also, the control group did not include individuals with a chronic medical condition or surgery in infancy or early childhood. The presence of a chronic medical condition has been demonstrated to have an impact on psychological wellbeing [30]. However, the control group consisted of siblings who were chosen for this group because they would have had similar life experiences as the cohort studied. Due to the rarity of the disorders, we were hampered by a small sample size. This did not allow us to do disorder-specific analysis. The results from this study are also derived from a survey completed by the participants' parents. Jürgensen et al. [22] demonstrated in their study that there is a discrepancy between the results gained from the patients as compared to their parents, whereby the parents overestimate their children's physical wellbeing but underestimate their emotional wellbeing. In view of the fact that the perception of the adequateness of quality of life will be dependent on who evaluates it, it is important that the voice of the children as the primary stakeholders should be heard. Therefore, future studies will need to evaluate the children's concerns and perceptions of quality of life.

The findings of this study highlight the fact that this group of patients needs to be studied more fully, especially given the diversity of the diagnoses and the complexities of management. This also lends support to the proposal that a skilled multidisciplinary team is necessary to manage these patients and answer their concerns [10, 31]. Surgical repair should also be carried out in designated centres of excellence to improve long-term outcomes for patients [30]. The way forward would involve compiling documentation consisting of the medical data and outcomes of this group of patients from childhood to adolescence in a nationwide database to allow systematic recording of information, an approach that was proposed by the multidisciplinary meeting on DSD held in Annecy, France, in 2012 [5].

It is also necessary that we conduct detailed studies on patients with a specific diagnosis by using qualitative research tools if required [32]. Given the trend in recent years to increasingly preserve patient autonomy, we also need to evaluate management principles in line with our sociocultural and religious framework [33]. The complexity of managing patients with DSD in a culture that is strongly male dominated and where the role of women is still seen as one of bearing and bringing up children needs 
to be addressed [26]. Thus, gender assignment and management of these patients must take into account the family's sociocultural and religious belief system in addition to the pathophysiology of the disorder $[34,35]$. However, it is worth remembering that the framework for decisionmaking should be based on ethical principles that can transcend all cultural and social mores [36]. Ultimately, we hope that through such efforts our management principles will be guided by robust scientific principles and will ultimately result in better care for all patients with DSD.

\section{Acknowledgement}

The authors would like to thank the patients and their parents who very kindly participated in this study.

\section{Disclosure Statement}

The authors have no conflicts of interest to disclose.

\section{References}

$>1$ Lee PA, Nordenstrom A, Houk CP, Ahmed SF, Auchus R, Baratz A, et al: Global disorders of sex development update since 2006: perceptions, approach and care. Horm Res Paediatr 2016;85:158-180.

$\checkmark 2$ Ismail SI, Mazen IA: A study of gender outcome of Egyptian patients with 46,XY disorder of sex development. Sex Dev 2010;4:285291.

$>3$ Wiesemann C, Ude-Koeller S, Sinnecker GH, Thyen U: Ethical principles and recommendations for the medical management of differences of sex development (DSD)/intersex in children and adolescents. Eur J Pediatr 2010; 169:671-679.

4 Hughes LA, Nihoul-Fekete C, Thomas B, Cohen-Kettenis PT: Consequences of the ESPE/ LWPES guidelines for diagnosis and treatment of disorders of sex development. Best Pract Res Clin Endocrinol Metab 2007;21: 351-365.

5 Schober J, Nordenstrom A, Hoebeke P, Lee P, Houk C, Looijenga L, et al: Disorders of sex development: summaries of long-term outcome studies. J Pediatr Urol 2012;8:616-623.

6 Creighton SM: Long-term outcome of feminization surgery: the London experience. BJU Int 2004;93(suppl 3):44-46.

7 Mazur T: Gender dysphoria and gender change in androgen insensitivity or micropenis. Arch Sex Behav 2005;34:411-421.

$>8$ Reiner WG: Gender identity and sex-of-rearing in children with disorders of sexual differentiation. J Pediatr Endocrinol Metab 2005; 18:549-553.

$>9$ The World Health Organization Quality of Life assessment (WHOQOL): Position paper from the World Health Organization. Soc Sci Med 1995;41:1403-1409.

-10 Amaral RC, Inacio M, Brito VN, Bachega TA, Domenice S, Arnhold IJ, et al: Quality of life of patients with 46,XX and 46,XY disorders of sex development. Clin Endocrinol 2015;82: 159-164.
11 Wisniewski AB, Mazur T: 46,XY DSD with female or ambiguous external genitalia at birth due to androgen insensitivity syndrome, 5alpha-reductase-2 deficiency, or 17beta-hydroxysteroid dehydrogenase deficiency: a review of quality of life outcomes. Int J Pediatr Endocrinol 2009;2009:567430.

12 Kuhnle U, Krahl W: The impact of culture on sex assignment and gender development in intersex patients. Perspect Biology and Med 2002;45:85-103.

13 Nimkarn S, Likitmaskul S, Sangacharoenkit P, Pathomvanich A, Sawathiparnich P, Wacharasindhu S, et al: Ambiguous genitalia: an overview of 22 years experience and the diagnostic approach in the Pediatric Department, Siriraj Hospital. J Med Assoc Thai 2002; 85(suppl 2):S496-S505.

14 Gupta DK, Menon PS: Ambiguous genitalia - an Indian perspective. Ind J Pediatr 1997; 64:189-194.

15 Lux A, Kropf S, Kleinemeier E, Jürgensen M, Thyen U; DSD Network Working Group: Clinical evaluation study of the German network of disorders of sex development (DSD)/ intersexuality: study design, description of the study population, and data quality. BMC Public Health 2009;9:110.

16 Houk CP, Lee PA: Update on disorders of sex development. Curr Opin Endocrinol Diabet Obes 2012;19:28-32.

17 Varni JW, Seid M, Rode CA: The PedsQL: measurement model for the pediatric quality of life inventory. Med Care 1999;37:126-139.

18 Varni JW, Seid M, Kurtin PS: PedsQL 4.0: reliability and validity of the Pediatric Quality of Life Inventory version 4.0 generic core scales in healthy and patient populations. Med Care 2001;39:800-812.

19 Palermo TM, Long AC, Lewandowski AS, Drotar D, Quittner AL, Walker LS: Evidencebased assessment of health-related quality of life and functional impairment in pediatric psychology. J Pediatr Psychol 2008;33:983996; discussion 997-998.
20 Ravens-Sieberer U, Erhart M, Wille N, Wetzel R, Nickel J, Bullinger M: Generic health-related quality-of-life assessment in children and adolescents: methodological considerations. Pharmacoeconomics 2006;24:1199-1220.

21 Johannsen TH, Ripa CP, Mortensen EL, Main KM: Quality of life in 70 women with disorders of sex development. Eur J Endocrinol 2006;155:877-885.

22 Jürgensen M, Lux A, Wien SB, Kleinemeier E, Hiort O, Thyen U: Health-related quality of life in children with disorders of sex development (DSD). Eur J Pediatr 2014;173:893-903.

23 Wisniewski AB: Gender development in 46,XY DSD: influences of chromosomes, hormones, and interactions with parents and healthcare professionals. Scientifica 2012; 2012:834967.

24 Lee PA, Houk CP: Long-term outcome and adjustment among patients with DSD born with testicular differentiation and masculinized external genital genitalia. Pediatr Endocrinol Rev 2012;10:140-151.

25 Zainuddin AA, Grover SR, Shamsuddin K, Mahdy ZA: Research on quality of life in female patients with congenital adrenal hyperplasia and issues in developing nations. J Pediatr Adolesc Gynecol 2013;26:296-304.

26 Ediati A, Juniarto AZ, Birnie E, Drop SL, Faradz SM, Dessens AB: Body image and sexuality in Indonesian adults with a disorder of sex development (DSD). J Sex Res 2015;52:15-29.

27 Minto CL, Liao LM, Woodhouse CR, Ransley PG, Creighton SM: The effect of clitoral surgery on sexual outcome in individuals who have intersex conditions with ambiguous genitalia: a cross-sectional study. Lancet 2003; 361:1252-1257.

28 Schonbucher V, Schweizer K, Rustige L, Schutzmann K, Brunner F, Richter-Appelt H: Sexual quality of life of individuals with $46, \mathrm{XY}$ disorders of sex development. J Sex Med 2012; 9:3154-3170 
29 Crowley R, Wolfe I, Lock K, McKee M: Improving the transition between paediatric and adult healthcare: a systematic review. Arch Dis Child 2011;96:548-553.

- 30 Warne G, Grover S, Hutson J, Sinclair A, Metcalfe S, Northam E, et al: A long-term outcome study of intersex conditions. J Pediatr Endocrinol Metab 2005;18:555-567.

31 Cohen-Kettenis PT: Psychosocial and psychosexual aspects of disorders of sex development. Best Pract Res Clin Endocrinol Metab 2010;24:325-334.
32 Kogan BA, Gardner M, Alpern AN, Cohen LM, Grimley MB, Quittner AL, et al: Challenges of disorders of sex development: diverse perceptions across stakeholders. Horm Res Paediatr 2012;78:40-46.

33 Lang C, Kuhnle U: Intersexuality and alternative gender categories in non-Western cultures. Horm Res 2008;69:240-250.

34 Julka S, Bhatia V, Singh U, Northam E, Dabadghao P, Phadke S, et al: Quality of life and gender role behavior in disorders of sexual differentiation in India. J Pediatr Endocrinol Metab 2006;19:879-888.
35 Warne GL, Raza J: Disorders of sex development (DSDs), their presentation and management in different cultures. Rev Endocr Metab Disord 2008;9:227-236.

-36 Mieszczak J, Houk CP, Lee PA: Assignment of the sex of rearing in the neonate with a disorder of sex development. Curr Opin Pediatr 2009;21:541-547. 